\title{
Towards Sub-Angström Ptychographic Diffractive Imaging
}

\author{
P. Wang ${ }^{1}$, D. J. Batey ${ }^{2}$, J. M. Rodenburg ${ }^{2}$, H. Sawada ${ }^{3}$ and A. I. Kirkland ${ }^{4}$
}

1. National Laboratory of Solid State Microstructures and College of Engineering and Applied sciences, Nanjing University, Nanjing 210093, People's Republic of China

2.Department of Electronic \& Electrical Engineering, University of Sheffield, Mappin Street, Sheffield, S1 3JD, UK

3. JEOL Ltd, 1-2 Mushashino, 3-Chome, Akishima, Tokyo 196, Japan.

4. Department of Materials, University of Oxford, Parks Road, Oxford OX1 3PH, UK

Transmission electron microscopy (TEM) is an established tool for characterizing the structure and chemistry of nano-materials. Spherical aberration correction has enabled sub-Angström image resolution to be achieved in the TEM at intermediate voltages[1]. Although this is a very significant achievement, the resolution is still ca. 25 times poorer than the theoretical diffraction limit and is limited by residual uncorrected aberrations in the magnetic objective lens. In addition, conventional TEM or Scanning TEM (STEM) imaging modes do not directly recover the complex electron wave front at the exit surface of the specimen. Focal or tilt azimuth series reconstruction[2] and Gabor holography[3] overcome this latter limitation by recovering the complex specimen exit surface wavefunction but the achievable resolution is still electron optically restricted.

An alternative approach is electron Coherent Diffractive Imaging (CDI)[4], which can be used to solve the phase problem from a single diffraction pattern by computationally iterating between the diffraction and object planes. Conventional CDI however, requires a priori knowledge about the shape or extent of the object. Rodenburg has suggested ptychographic electron coherent diffractive imaging (PE-CDI), implemented using a ptychographical iterative engine (PIE)[5], in which the illumination function is moved over a sample while multiple diffraction patterns are recorded from overlapping areas. PE-CDI records redundant information from overlapping illumination positionsand enables PIE to potentiallyovercome many of the issues (i.e. non-unique solutions, limited field of view etc) that affect conventional CDI, even without a prior information about the object. As this approach is diffraction limited, it can, in principle, overcome current image resolution limiting factors and ultimately achieve wavelength-limited resolution. Recently both Putkunz, et al.[6] and Humphry, et al.[7] have demonstrated that this technique can achieve a resolution beyond that obtained in the corresponding HAADF images recorded using the same probe. However, resolutions comparable to those achieved using aberration corrected optics have not yet been demonstrated.

The PIE reconstruction algorithm assumes that the object is a thin 2D grating with uniform thickness.For this type of sample, PIE can provide a solution for an illumination function that is consistent across one plane of the sample. In reality however, many TEM samples exhibit a thickness variation and for a wedge shaped sample the illumination function changes (i.e. defocus value) as a function of thickness. In this contribution we investigate the effect of the thickness variation of the specimen during restoration usingthe PIE algorithm. The sample used was a $\mathrm{CeO}_{2}$ nanoparticle over hanging a hole in a carbon film. These particleshave atruncated octahedral morphology, with a well defined thickness along the optical axis. A probe-forming aperture with a semi-angle of $25 \mathrm{mrad}$ was used to create a STEM probe with a large defocus at the specimen. This illumination function was scanned across the area of interest in a 5x5 array indicated by a white box shown in Fig 1a). Multiple far-field diffraction patterns were recorded using a JEOL R005 $300 \mathrm{kV}$ aberration corrected 
FEGTEM. Fig. 1 b) shows a typical recorded electron diffraction pattern and Fig. 1 c) shows the initial probe function used in the reconstruction estimated form the full set of measured aberration coefficients. The reconstruction algorithm used here is that described in Reference[8]. Fig. 2 a) shows the HAADF image of the same particle. In conclusion, we present that a reconstruction of the object in both amplitude and phase with atomic resolution as shown in Figs. 2 b) and c) and demonstrate that this technique provides a fast, direct and deterministic approach to evaluating surface atomic structures on the terminal atomic (surface) layer.

\section{References}

[1] PD Nellist et al., Science 305 (2004) p.1741.

[2] RR Meyer et al., Ultramicroscopy92 (2002) p.89-109.

[3] H Lichte et al., Annual Review of Materials Research 37 (2007) p.539-588.

[4] RW Gerchberg and O Saxton, Optik35 (1972) p.237-246.

[5] JM Rodenburg and HML Faulkner, Applied Physics Letters 85 (2004) p.4795-4797.

[6] CT Putkunz et al., Physical Review Letters 108 (2012) p.073901.

[7] MJ Humphry et al., Nat Commun3 (2012) p.730.

[8] AM Maiden and JM Rodenburg, Ultramicroscopy109 (2009) p.1256-1262.

[9] The authors gratefully acknowledge funding from the EPSRC Basic Technology Grant under No. EP/E034055/1 and Zhongyang Grant.
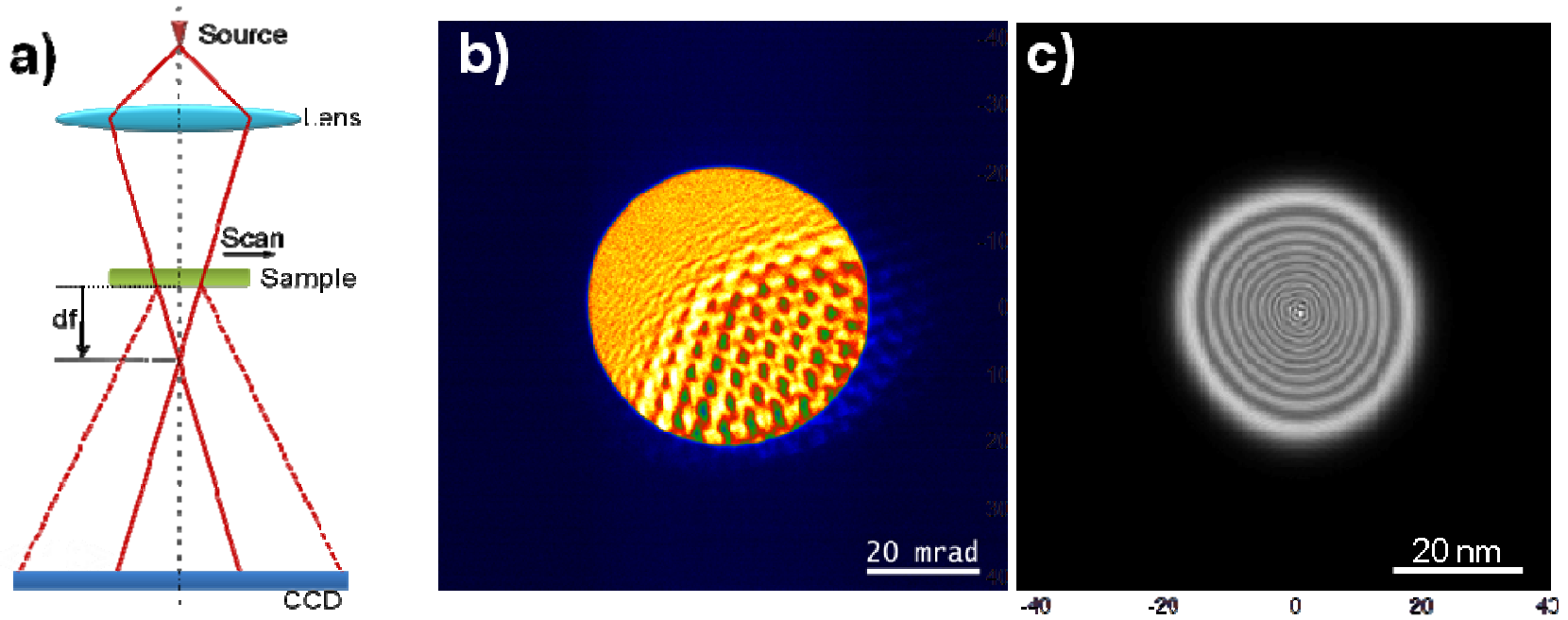

Figure 1.a) Schematic diagrams of the experimental configuration. b) A typical diffraction pattern recorded on the CCD. c) The intensity of the initial probe function.
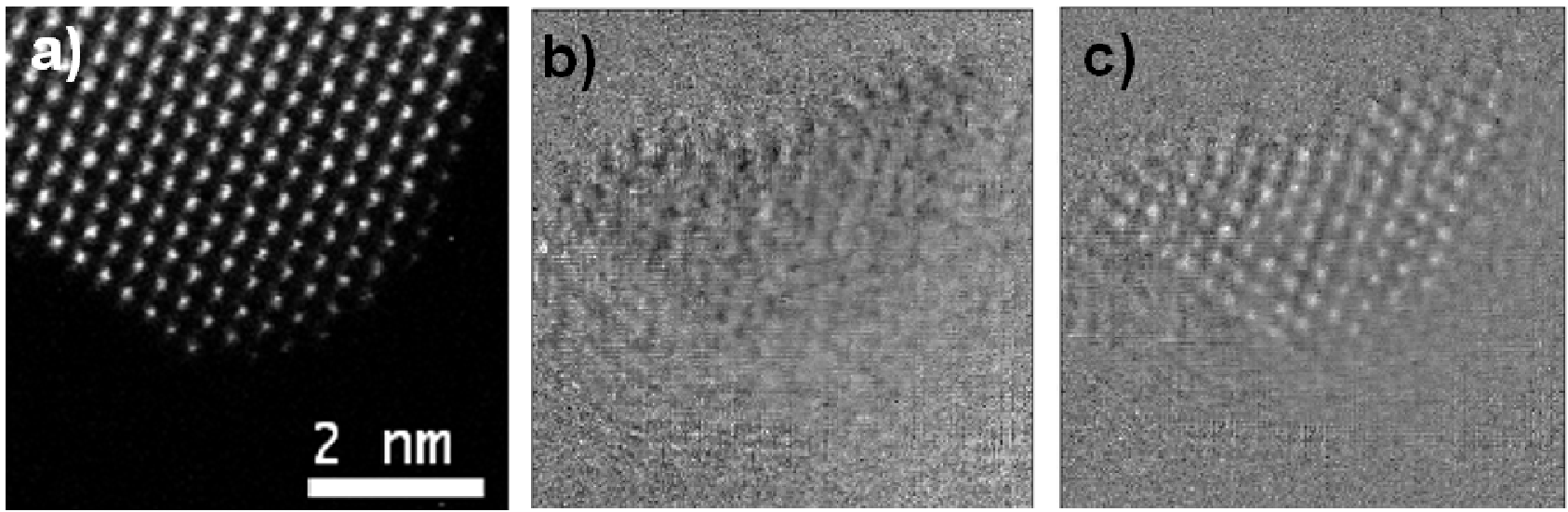

Figure 2. a) STEM-HAADF image from the tip of a $<110>\mathrm{CeO}_{2}$ particle; b) and c) amplitude and phase of construction of the same $\mathrm{CeO}_{2}$ particle, respectively. 